\title{
Minimum-Power Multicast Routing in Static Ad Hoc Wireless Networks
}

\author{
Peng-Jun Wan, Gruia Călinescu, and Chih-Wei Yi
}

\begin{abstract}
Wieselthier et al. (2000) proposed three greedy heuristics for Min-Power Asymmetric Broadcast Routing: SPT (shortest-path tree), MST (minimum spanning tree), and BIP (broadcasting incremental power). Wan et al. (2001) proved that SPT has an approximation ratio of at least $(n / 2)$ where $n$ is the total number of nodes, and both MST and BIP have constant approximation ratios. Based on the approach of pruning, Wieselthier et al. also proposed three greedy heuristics for Min-Power Asymmetric Multicast Routing: P-SPT (pruned shortest-path tree), P-MST (pruned minimum spanning tree), and P-BIP (pruned broadcasting incremental power). In this paper, we first prove that the approximation ratios of these three heuristics are at least $(n-1 / 2), n-1$, and $n-2-o(1)$, respectively. We then present constant-approxiation algorithms for Min-Power Asymmetric Multicast Routing. We show that any $\rho$-approximation Steiner tree algorithm gives rise to a $c \rho$-approximation heuristic for Min-Power Asymmetric Multicast Routing, where $c$ is a constant between 6 and 12. In particular, the Takahashi-Matsuyama Steiner tree heuristic leads to a heuristic called SPF (shortest-path first), which has an approximation ratio of at most $2 c$. We also present another heuristic, called MIPF (minimum incremental path first), for Min-Power Asymmetric Multicast Routing and show that its approximation ratio is between $(13 / 3)$ and $2 c$. Both SPF and MIPF can be regarded as an adaptation of MST and BIP, respectively, in a different manner than pruning. Finally, we prove that any $\rho$-approximation Steiner tree algorithm also gives rise to a $2 \rho$-approximation algorithm for Min-Power Symmetric Multicast Routing.
\end{abstract}

Index Terms-Approximation algorithms, multicast routing, power control.

\section{INTRODUCTION}

$\mathbf{T}$ HE RECENT advances in the development of affordable and portable wireless communication and computation devices has fostered a tremendous amount of research on ad hoc wireless networks. Ad hoc networks can be used wherever a fixed backbone infrastructure is not viable, e.g., in battlefield and emergency disaster relief. Typically, omnidirectional antennas are used by all nodes to transmit and receive signals. Thus, a transmission made by a node can be received by all nodes within its transmission range. A communication session is achieved either through single-hop transmission if the recipient is within the transmission range of the source node, or by relaying through intermediate nodes otherwise. For this reason, ad hoc wireless networks are also called multihop packet radio networks.

Manuscript received August 1, 2000; revised August 16, 2002; approved by IEEE/ACM TRANSACTIONS ON NETWORKING Editor M. Ammar.

The authors are with the Department of Computer Science, Illinois Institute of Technology, Chicago, IL 60616 (e-mail: wan@cs.iit.edu; calinescu@cs.iit.edu; yichihw@iit.edu).

Digital Object Identifier 10.1109/TNET.2004.828940
One of the major concerns in ad hoc wireless networks is reducing node energy consumption. In fact, nodes are usually powered by batteries of limited capacity. Once the nodes are deployed, it is very difficult or even impossible to recharge or replace their batteries in many application scenarios. Hence, reducing power consumption is often the only way to extend network lifetime. As demonstrated by [7], the power consumption is dominated by communications. For the purpose of energy conservation, each node can (possibly dynamically) adjust its transmitting power, based on the distance to the receiving node and the background noise. In the most common power-attenuation model [10], the signal power falls as $\left(1 / d^{\kappa}\right)$ where $d$ is the Euclidean distance from the transmitter antenna and $\kappa$ is the pass-loss exponent of the wireless environment-a real constant typically between 2 and 5. Assume that all receivers have the same power threshold for signal detection, which is typically normalized to one. Then, the power required to cover a transmission range of radius $d$ is $d^{\kappa}$.

Due to the nonlinear power attenuation, the total power consumption required by a communication session can be potentially reduced by relaying signal through intermediate nodes [12], [16]. We assume throughout this paper that the set $V$ of nodes in a given wireless ad hoc network are located in a two-dimensional plane. A communication session is specified by a pair $(s, D)$ where $s \in V$ is the source and $D \subseteq V \backslash\{s\}$ is the set of destinations. If $D$ consists of a single node, the communication session is a unicast. If $D$ consists of all nodes other than the source $s$, the session is a broadcast. For general $D$, the communication session is a multicast. Depending on whether the communications is unidirectional or bidirectional, there are two variations on routing and power consumptions.

\section{A. Min-Power Asymmetric Routing}

In the case of unidirectional communications, a routing for a communication session $(s, D)$ is an arborescence (a directed tree) $T$ rooted at $s$ which reaches all nodes in $D$. An arborescence $T$ determines the transmission power of every node in $T$ as follows: the transmission power of each sink is zero, and the transmission power of each node $v$ of $T$ other than sinks is equal to the $\kappa$ th power of the longest distance between $u$ and its children in $T$. The total power required by $T$ is then the sum of the transmission power of all nodes in $T$. The problem Min-Power Asymmetric Routing then seeks, for any given communication session $(s, D)$, an arborescence of minimum total power which is rooted at $s$ and reaches all nodes in $D$.

There are two special cases of Min-Power Asymmetric Routing. The special case in which all communication sessions are restricted to unicasts is referred to as Min-Power 
Asymmetric Unicast Routing, and the special case in which all communication sessions are restricted to broadcasts is referred to as Min-Power Asymmetric Broadcast Routing. While the former is easily solved in polynomial time by shortest-path algorithms, the latter is NP-hard as shown by Clementi et al. [5]. Wieselthier et al. [16] presented three greedy broadcasting heuristics, namely MST (minimum spanning tree), SPT (shortest-path tree), and BIP (broadcasting incremental power), and evaluated them by simulations. Wan et al. [15] provided a theoretical analysis on the approximation ratios of these three heuristics. They showed that MST has an approximation ratio between 6 and 12, BIP has an approximation ratio between $(14 / 3)$ and 12 , and SPT has an approximation ratio as large as $(n / 2)-o(1)$, where $n$ is the number of nodes.

Min-Power Asymmetric Multicast Routing is the most general form of Min-Power Asymmetric Routing where the communication sessions can be arbitrary multicasts. As a generalization of Min-Power Asymmetric Broadcast Routing, Min-Power Asymmetric Multicast Routing is also NP-hard. Wieselthier et al. [16] adapted their three broadcasting heuristics to three multicasting heuristics by a technique of pruning, which we refer to as pruned minimum spanning tree (P-MST), pruned shortest-path tree (P-SPT), and pruned broadcasting incremental power (P-BIP), respectively. The idea is to first obtain a spanning arborescence rooted at the source of a given multicast session by applying any of the three broadcasting heuristics, and then eliminate from the spanning arborescence all nodes which do not have any descendant in the given multicast session.

Wieselthier et al. [16] had evaluated their three multicasting heuristics through simulations, but their performance in terms of approximation ratios remained unsolved. The analysis in [15] on the performance of SPT can be extended to P-SPT, which leads to the same lower bound on the approximation ratio of P-SPT. However, the analysis in [15] on the constant approximation ratios of MST and BIP cannot be extended P-MST and P-BIP. One might expect that both P-MST and P-BIP have the same approximation ratios as their broadcasting counterparts. Surprisingly, as will be demonstrated in this paper, neither P-MST nor P-BIP has constant approximation ratio. We show that P-MST has an approximation ratio of at least $n-1$, and P-BIP has an approximation ratio of at least $n-2-o(1)$.

Since none of the three heuristics proposed by Wieselthier et al. [16] have constant approximation ratios, we are motivated to address the existence of heuristics with constant approximation ratio. We first prove that any heuristic of approximation ratio $\rho$ for Steiner minimum tree (SMT) [8], [9] in graphs gives rise to a heuristic for Minimum-Energy Asymmetric Multicast Routing of approximation ratio at most $c \rho$, where $c$ is a constant between 6 and 12. In particular, the Robins-Zelikovsky Steiner tree heuristic [11] leads to an asymmetric multicasting heuristic of approximation ratio approaching $c(1+(\ln 3 / 2))$, and the Takahashi-Matsuyama Steiner tree heuristic [14] leads to an asymmetric multicasting heuristic called SPF (shortest path first), which has an approximation ratio of at most $2 c$. After that, we present another asymmetric multicasting heuristic, called MIPF (minimum incremental path first), and show that its approximation ratio $\backslash$ is between (13/3) and $2 c$. Both SPF and MIPF can be regarded as an adaptation of MST and BIP, respectively, in a different manner than pruning.

\section{B. Min-Power Symmetric Routing}

In the case of bidirectional communications, a routing for a communication session $(s, D)$ is a Steiner tree $T$ for $\{s\} \cup D$. A Steiner tree $T$ determines the transmission power of every node in $T$ : the transmission power of each node $v$ of $T$ is equal to the $\kappa$ th power of the longest distance between $u$ and its neighbors in $T$. The total power required by $T$ is then the sum of the transmission power of all nodes in $T$. The problem Min-Power Symmetric Routing then seeks, for any given communication session $(s, D)$, a Steiner tree for $\{s\} \cup D$ of minimum total power.

Similar to their asymmetric counterparts, Min-Power Symmetric Unicast Routing can be solved in polynomial time by shortest-path algorithms as shown by Calinescu et al. [3], and Min-Power Symmetric Broadcast Routing is NP-hard as proven by Blough et al. [2]. An earlier heuristic developed by Chen and Huang [4] based on minimum spanning tree is a 2-approximation for the Min-Power Symmetric Broadcast Routing even with arbitrary (i.e., not necessarily Euclidean) distance between any pair of nodes. Calinescu et al. [3] establish the similarity between Min-Power Symmetric Broadcast Routing and the classic Steiner tree problem in graphs, give a polynomial-time approximation algorithm with approximation ratio approaching $1+$ $\ln 2 \approx 1.69$, and present a more practical 15/8-approximation algorithm. To our best knowledge, Min-Power Symmetric Multicast Routing has not been addressed yet. In this paper, we prove that any heuristic of approximation ratio $\rho$ for Steiner minimum tree in graphs gives rise to a heuristic of approximation ratio at most $2 \rho$ for Min-Power Symmetric Multicast Routing.

The remaining of this paper is organized as follows. In Section II, we prove the $\Omega(n)$ lower bounds on the approximation ratios of the three asymmetric multicasting heuristics, P-SPT, P-MST, and P-BIP proposed by Wieselthier et al. [16]. In Section III, we present constant-approximation algorithms for both asymmetric multicasting and symmetric multicasting. Finally, in Section IV we summarize this paper.

\section{PRuning Is Not GoOD ENOUGH}

Wieselthier et al. [16] presented three heuristics for Min-Power Asymmetric Multicast Routing. All three heuristics obtain a multicast arborescence by pruning a spanning arborescence rooted at the source of the multicast session. The spanning arborescence is constructed by one of their three heuristics: minimum spanning tree (MST), shortest-path tree (SPT), and broadcasting incremental power (BIP) for Min-Power Asymmetric Broadcast Routing. The MST heuristic first applies the Prim's algorithm [6] to obtain an MST, and then orient it as an arborescence rooted at the source node. The SPT heuristic first applies the Dijkstra's algorithm [6] to obtain a shortest-path tree from the source node, and then orient the shortest-path tree as an arborescence rooted at the source node. The BIP heuristic is the node version of Dijkstra's algorithm for shortest-path tree. It maintains throughout its execution a single arborescence rooted at the source node. The arborescence starts from the 


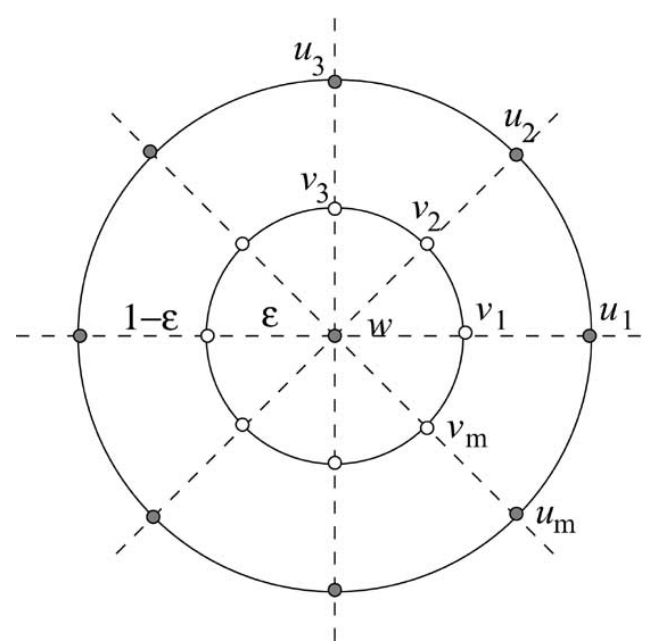

Fig. 1. A bad instance for P-SPT.

source node, and new nodes are added to the arborescence one at a time on a minimum incremental cost basis until all nodes are included in the arborescence. The incremental cost of adding a new node is the minimum additional power increased by some node in the current arborescence so as to reach this new node. Corresponding to MST, SPT, and BIP, the three heuristics for Min-Power Asymmetric Multicast Routing are referred to as pruned minimum spanning tree (P-MST), pruned shortest-path tree (P-SPT), and pruned broadcasting incremental power (P-BIP), respectively.

In this section, we show that the approximation ratios of these three heuristics are all at least as large as $\Omega(n)$, where $n$ is the number of nodes in the network. We begin with the P-SPT algorithm.

Lemma 1: The approximation ratio of P-SPT is at least $((n-1) / 2)$.

Proof: Let $\epsilon$ be a sufficiently small positive number. Consider $m$ nodes $u_{1}, u_{2}, \ldots, u_{m}$ evenly distributed on a circle of radius 1 centered at node $w$ (see Fig. 1). For $1 \leq i \leq m$, let $v_{i}$ be the point in the line segment $w u_{i}$ with $\left\|w v_{i}\right\|=\epsilon$. We consider a network consisting of $n=2 m+1$ nodes

$$
w, u_{1}, u_{2}, \ldots, u_{m}, v_{1}, v_{2}, \ldots, v_{m},
$$

and a multicast session from node $w$ to the $m$ nodes $u_{1}, u_{2}, \ldots, u_{m}$. The shortest-path tree is the superposition of the paths $w v_{i} u_{i}$. Since no node can be removed from this shortest-path tree, it is also the output by P-SPT. Its total power is

$$
\epsilon^{2}+m(1-\epsilon)^{2}
$$

On the other hand, if the transmission power of node $w$ is 1 , then the signal can reach all points $u_{1}, u_{2}, \ldots, u_{m}$. Thus, the minimum power is at most 1 . So the approximation ratio of P-SPT is at least $\epsilon^{2}+m(1-\epsilon)^{2}$. As $\epsilon \rightarrow 0$, this ratio converges to $m=((n-1) / 2)$.

Next, we construct bad instances for P-MST.

Lemma 2: The approximation ratio of P-MST is at least $n-1$.

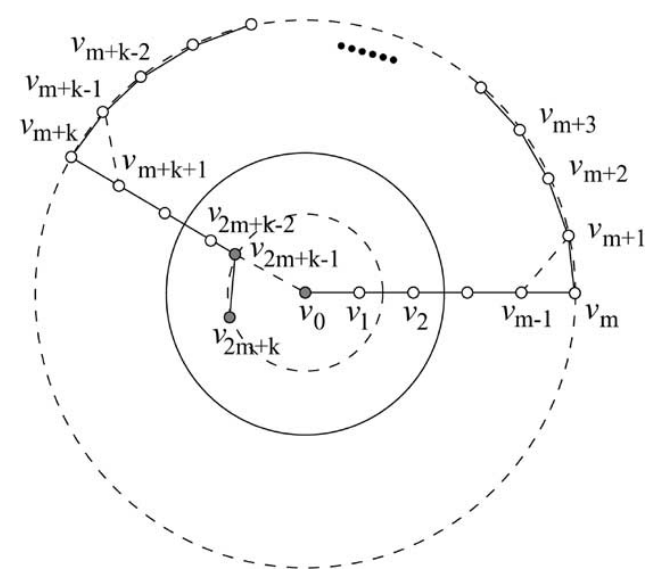

Fig. 2. A bad instance for P-MST.

Proof: Fix a circle of radius 1 centered at node $v_{0}$ (see Fig. 2), and choose a sufficiently large integer $m$. Let $v_{1}, \ldots, v_{m}$ be the $m$ points on a radius of the circle with

$$
\left\|v_{i} v_{i+1}\right\|=\frac{1}{m}
$$

for $1 \leq i<m$. Then $v_{m}$ is on the circle. Let $v_{m+1}, \ldots, v_{m+k}$ be the $k$ points distributed on the circle counterclockwise with

$$
\left\|v_{i} v_{i+1}\right\|=\frac{1}{m}
$$

for all $m \leq i<m+k$ and the angle $v_{m} v_{0} v_{m+k}$ is between $(\pi / 2)$ and $\pi$. Let $\epsilon$ be a sufficiently small positive number which is less than $(1 / m)$. Let $v_{m+k+1}, \ldots, v_{2 m+k-1}$ be the $m-1$ points on the radius $v_{0} v_{m+k}$ with

$$
\left\|v_{i} v_{i+1}\right\|=\frac{1}{m}
$$

for $m+k \leq i<2 m+k-2$ and

$$
\left\|v_{2 m+k-2} v_{2 m+k-1}\right\|=\frac{1}{m}-\epsilon \text {. }
$$

Then

$$
\left\|v_{0} v_{2 m+k-1}\right\|=\frac{1}{m}+\epsilon .
$$

Finally, let $v_{2 m+k}$ be the node lying on the circle of radius $(1 / m)+\epsilon$ centered at node $v_{0}$ with

$$
\left\|v_{2 m+k-1} v_{2 m+k}\right\|=\frac{1}{m} \text {. }
$$

Now we consider the network consisting of these $n=2 m+k+1$ nodes and a multicast session from node $v_{0}$ to the two nodes $v_{2 m+k-1}$ and $v_{2 m+k}$. As the angle $v_{m-1} v_{m} v_{m+1}$ is greater than $(\pi / 3)$, we have

$$
\left\|v_{m-1} v_{m+1}\right\|>\left\|v_{m-1} v_{m}\right\|=\left\|v_{m} v_{m+1}\right\|=\frac{1}{m} .
$$

Similarly

$$
\left\|v_{m+k-1} v_{m+k+1}\right\|>\frac{1}{m} .
$$

Thus, the execution of Prim's algorithm for MST outputs the path

$$
\begin{aligned}
& v_{0} v_{1} \ldots v_{m-1} v_{m} v_{m+1} \ldots v_{m+k-1} v_{m+k} \\
& v_{m+k+1} \ldots v_{2 m+k-2} v_{2 m+k-1} v_{2 m+k}
\end{aligned}
$$




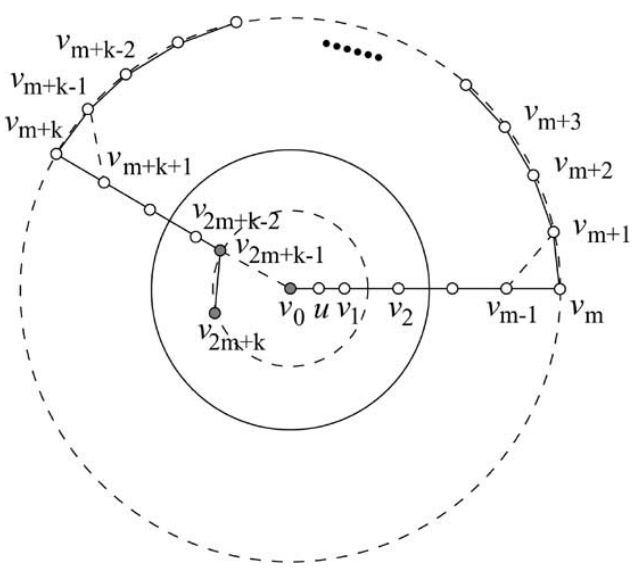

Fig. 3. A bad instance for P-BIP.

as the MST. Notice that except the link $v_{2 m+k-2} v_{2 m+k-1}$ which has length of $(1 / m)-\epsilon$, all other $2 m+k-1$ links have length of $(1 / m)$. In addition, no node can be pruned from this path. Thus, this path is output by P-MST. Its total power consumption is

$$
(2 m+k-1) \frac{1}{m^{2}}+\left(\frac{1}{m}-\epsilon\right)^{2} .
$$

On the other hand, a direct transmission from node $v_{0}$ at the power of $((1 / m)+\epsilon)^{2}$ can reach the two destination nodes $v_{2 m+k-1}$ and $v_{2 m+k}$. Thus, the minimum power is at most $((1 / m)+\epsilon)^{2}$. Therefore, the approximation ratio of P-MST is at least

$$
\frac{(2 m+k-1) \frac{1}{m^{2}}+\left(\frac{1}{m}-\epsilon\right)^{2}}{\left(\frac{1}{m}+\epsilon\right)^{2}} .
$$

As $\epsilon \rightarrow 0$, this ratio converges to $2 m+k=n-1$.

Finally, we construct bad instances for P-BIP. Its construction is similar to that in the above proof but the analysis is more subtle.

Lemma 3: The approximation ratio of P-BIP is at least $n-2-o(1)$.

Proof: In the instance constructed in the Proof of Lemma 3, we add a point $u$ in the line segment $v_{0} v_{1}$ with $\left\|v_{0} u\right\|=\epsilon$. Then $\left\|u v_{1}\right\|=(1 / m)-\epsilon$. We consider the network consisting of these $n=2 m+k+2$ nodes and a multicast session from node $v_{0}$ to the two nodes $v_{2 m+k-1}$ and $v_{2 m+k}$.

Let us first examine the execution of the BIP algorithm. Initially, the arborescence consists of only $v_{0}$. The first node to be added is $u$ by increasing the transmission power of node $v_{0}$ from 0 to $\epsilon^{2}$. The incremental power to reach $v_{2 m+k-1}$ or $v_{2 m+k}$ from node $v_{0}$ is

$$
\left(\frac{1}{m}+\epsilon\right)^{2}-\epsilon^{2}=\frac{1}{m^{2}}+\frac{2 \epsilon}{m}>\frac{1}{m^{2}} .
$$

Thus, the second node to be added is $v_{1}$ by increasing of the transmission power of node $u$ from 0 to $((1 / m)-\epsilon)^{2}$. Subsequently, the $m-1$ nodes $v_{2}, \ldots, v_{m}$ are added sequentially and the $m-1$ nodes $v_{1}, \ldots, v_{m-1}$ all transmit at the power level of $\left(1 / \mathrm{m}^{2}\right)$. Notice that the incremental power to add nodes $v_{2 m+k-1}$ or $v_{2 m+k}$ is always $\left(1 / m^{2}\right)+(2 \epsilon / m)>\left(1 / m^{2}\right)$, and thus, neither $v_{2 m+k-1}$ nor $v_{2 m+k}$ can be added by this time. In the next step, node $v_{m+1}$ is added. However, it is reached not from node $v_{m}$ by increasing the transmission power of node $v_{m}$ from 0 to $\left(1 / m^{2}\right)$, but from node $v_{m-1}$ whose transmission power is increased from

$$
\left\|v_{m-1} v_{m}\right\|^{2}=\frac{1}{m^{2}}
$$

to

$$
\begin{aligned}
\left\|v_{m-1} v_{m+1}\right\|^{2} & =\frac{2}{m^{2}}\left(1-\cos \angle v_{m-1} v_{m} v_{m+1}\right) \\
& =\frac{2}{m^{2}}\left(1-\frac{1}{2 m}\right) \\
& =\frac{2}{m^{2}}-\frac{1}{m^{3}} .
\end{aligned}
$$

Thus, node $v_{m}$ does not relay the signal, and the incremental power to add node $v_{m+1}$ is

$$
\left(\frac{2}{m^{2}}-\frac{1}{m^{3}}\right)-\frac{1}{m^{2}}=\frac{1}{m^{2}}-\frac{1}{m^{3}} .
$$

At this moment, the arborescence consists of the path $v_{0} u v_{1} \ldots v_{m-1}$, with $v_{m-1}$ having nodes $v_{m}$ and $v_{m+1}$ as its children. Since both $\angle v_{m-2} v_{m-1} v_{m+2}$ and $\angle v_{m-1} v_{m+1} v_{m+2}$ are obtuse, the following step adds node $v_{m+2}$ by increasing the power of node $v_{m+1}$ from 0 to $\left(1 / \mathrm{m}^{2}\right)$. Subsequently, it is easy to show that the $k-2$ nodes $v_{m+3}, \ldots, v_{m+k}$ are added sequentially and the $k-1$ nodes $v_{m+2}, \ldots, v_{m+k-1}$ all transmit at the power level of $\left(1 / \mathrm{m}^{2}\right)$. Following the same argument as above, we can show that node $v_{m+k+1}$ is added in the next by increasing the transmission power of node $v_{m+k-1}$ from $\left(1 / m^{2}\right)$ to $\left(2 / m^{2}\right)-\left(1 / m^{3}\right)$, and after that node $v_{m+k+2}$ is added by increasing the transmission power of node $v_{m+k+1}$ from 0 to $\left(1 / m^{2}\right)$. Thus, node $v_{m+k}$ does not relay the signal at all. Then the $m-3$ nodes $v_{m+k+3}, \ldots, v_{2 m+k-1}$ are added sequentially. The transmission powers of the $m-4$ nodes $v_{m+k+2}, \ldots, v_{2 m+k-3}$ are all $\left(1 / m^{2}\right)$, and the transmission power of node $v_{2 m+k-2}$ is $((1 / m)-\epsilon)^{2}$. Finally since the angle $v_{2 m+k-2} v_{2 m+k-1} v_{2 m+k}$ is obtuse, node $v_{2 m+k}$ is added by increasing the power of node $v_{2 m+k-1}$ from 0 to $\left(1 / m^{2}\right)$.

In summary, the output arborescence is the superposition of the path

$$
\begin{aligned}
& v_{0} u v_{1} \ldots v_{m-1} v_{m+1} v_{m+2} \ldots v_{m+k-2} v_{m+k-1} \\
& v_{m+k+1} v_{m+k+2} \ldots v_{2 m+k-2} v_{2 m+k-1} v_{2 m+k}
\end{aligned}
$$

and two links $v_{m-1} v_{m}$ and $v_{m+k-1} v_{m+k}$. Note that only the two nodes $v_{m}$ and $v_{m+k}$ are eliminated during pruning and no node can decrease its transmission power after the pruning. The output P-BIP is exactly the above path, and its total power consumption is calculated below. Among all $n=2 m+k+2$ nodes:

- nodes $v_{m}, v_{m+k}$, and $v_{2 m+k}$ does not transmit at all;

- node $v_{0}$ has transmission power of $\epsilon^{2}$;

- nodes $u$ and $v_{2 m+k-2}$ have transmission power of $((1 / m)-\epsilon)^{2}$;

- nodes $v_{m-1}$ and $v_{m+k-1}$ have transmission power of $\left(2 / m^{2}\right)-\left(1 / m^{3}\right)$

- all other $n-8$ nodes have transmission power of $\left(1 / m^{2}\right)$. 
So the total power is

$$
\begin{aligned}
3 \cdot 0 & +1 \cdot \epsilon^{2}+2 \cdot\left(\frac{1}{m}-\epsilon\right)^{2} \\
+ & 2 \cdot\left(\frac{2}{m^{2}}-\frac{1}{m^{3}}\right)+(n-8) \cdot \frac{1}{m^{2}} \\
& =\frac{n-2}{m^{2}}-\frac{1}{m^{3}}-\frac{4 \epsilon}{m}+3 \epsilon^{2} .
\end{aligned}
$$

On the other hand, a direct transmission from node $v_{0}$ at the power of $((1 / m)+\epsilon)^{2}$ can reach the two destination nodes $v_{2 m+k-1}$ and $v_{2 m+k}$. Thus, the minimum power is at most $((1 / m)+\epsilon)^{2}$. Therefore, the approximation ratio of P-BIP is at least

$$
\frac{\frac{n-2}{m^{2}}-\frac{1}{m^{3}}-\frac{4 \epsilon}{m}+3 \epsilon^{2}}{\left(\frac{1}{m}+\epsilon\right)^{2}} .
$$

As $\epsilon \rightarrow 0$, this ratio converges to

$$
\frac{\frac{n-2}{m^{2}}-\frac{1}{m^{3}}}{\frac{1}{m^{2}}}=n-2-\frac{1}{m}=n-2-o(1) \text {. }
$$

\section{CONSTANT-APPROXIMATION AlgORITHMS}

In this section, we present constant-approximation algorithms for Min-Power Asymmetric Multicast Routing and for MinPower Symmetric Multicast Routing. In what follows, we use $V$ to denote the set of networking nodes that are located in a two-dimensional plane. We denote by $G$ the weighted complete graph over $V$ in which the weight of each edge $e$ is given by

$$
\omega(e) \equiv\|e\|^{\kappa}
$$

For any subgraph $H$ of $G$, the weight of $H$ is defined by

$$
\omega(H) \equiv \sum_{e \in E(H)} \omega(e) .
$$

\section{A. Asymmetric Multicast Routing}

For any finite point set $U$ in the two-dimensional plane, we use emst $(U)$ to denote an arbitrary Euclidean MST of $U$. Then the constant $c$ is defined by the supreme of $\sum_{e \in \operatorname{emst}(U)}\|e\|^{2}$ over all finite point sets $U$ located in a disk of radius one. It is proved in [15] that $6 \leq c \leq 12$. The following lemma presents an lower bound on the minimum power required by asymmetric multicasting of a given communication session.

Lemma 4: Any asymmetric routing for a communication session $(s, D)$ requires total power of at least $(1 / c)$ times the weight of the Steiner minimum tree for $\{s\} \cup D$.

Proof: Let OPT be a min-power arborescence for $(s, D)$ with total power opt. For any nonsink node $u$ in OPT, let $T_{u}$ be an Euclidean MST of the point set consisting $u$ and all children of $u$ in $T$. Suppose that the longest Euclidean distance between $u$ and its children is $r_{u}$. Then the transmission power of node $u$ is $r_{u}^{\kappa}$. Since all children of $u$ lie in the disk centered at $u$ with radius $r_{u}$, and the length of each edge in is at most $r_{u}$, by the definition of $c$ we have

$$
\sum_{e \in T_{u}}\left(\frac{\|e\|}{r_{u}}\right)^{\kappa} \leq \sum_{e \in T_{u}}\left(\frac{\|e\|}{r_{u}}\right)^{2} \leq c
$$

which implies that

$$
r_{u}^{\kappa} \geq \frac{1}{c} \sum_{e \in T_{u}}^{\kappa}\|e\|^{\kappa}=\frac{1}{c} \omega\left(T_{u}\right) .
$$

Let $\mathrm{OPT}^{\prime}$ denote the tree obtained by superposing of all $T_{u}$ 's for nonsink nodes $u$ of OPT. Then $\mathrm{OPT}^{\prime}$ is a Steiner tree for $\{s\} \cup D$. Since opt is equal to the summation of $r_{u}^{\kappa}$ over all nonsink nodes $u$ of OPT, we have

$$
\text { opt } \geq \frac{1}{c} \omega\left(\mathrm{OPT}^{\prime}\right)
$$

As $\omega\left(\mathrm{OPT}^{\prime}\right)$ is at least the weight of the Steiner minimum tree for $\{s\} \cup D$, the lemma follows.

Let $\mathcal{A}$ be any polynomial-time approximation algorithm for Steiner minimum tree in graphs. For any given communication session $(s, D)$, we first apply $\mathcal{A}$ on $G$ to obtain a Steiner tree for $\{s\} \cup D$, and then orient it to an arborescence rooted at the source, which is used for the asymmetric routing of $(s, D)$. We use $\mathcal{A}_{a}$ to denote this heuristic for Min-Power Asymmetric Multicast Routing.

Theorem 5: For any $\rho$-approximation algorithm $\mathcal{A}$ for Steiner minimum tree, the approximation ratio of the algorithm $\mathcal{A}_{a}$ is at most $c \rho$.

Proof: Fix a communication session $(s, D)$ and let opt be the minimum power required by its asymmetric routing. Let $T^{*}$ be a Steiner minimum tree for $\{s\} \cup D$ in $G$, and $T$ be the Steiner tree for $\{s\} \cup D$ in $G$ output by the algorithm $\mathcal{A}$. Then

$$
\omega(T) \leq \rho \cdot \omega\left(T^{*}\right)
$$

On the other hand, by Lemma 4

$$
\text { opt } \geq \frac{1}{c} \omega\left(T^{*}\right) \text {. }
$$

which implies that

$$
\omega\left(T^{*}\right) \leq c \cdot \text { opt }
$$

Thus

$$
\omega(T) \leq \rho \cdot \omega\left(T^{*}\right) \leq c \rho \cdot \mathrm{opt} .
$$

Since the total power required by the arborescence oriented from $T$ is at most $\omega(T)$, the theorem follows.

A number of constant-approximation algorithms for Steiner minimum trees in graphs have been proposed in literature [8], [9]. Robins and Zelikovsky [11] gave an approximation algorithm with the best known approximation ratio approaching $1+$ $(\ln 3 / 2) \approx 1.55$. Thus, it can give rise to a heuristic for MinPower Asymmetric Multicast Routing with approximation ratio approaching $c(1+(\ln 3 / 2)) \leq 12(1+(\ln 3 / 2)) \approx 18.6$. However, this heuristic may be not practical for ad hoc wireless networks due to its implementation complexity. 
Takahashi and Matsuyama [14] gave a simple 2-approximation algorithm for Steiner minimum tree in graphs. A heuristic for Min-Power Asymmetric Multicast Routing based on Takahashi-Matsuyama Steiner tree heuristic can be implemented easily as follows. Throughout the execution, we maintain an arborescence $T$ rooted at the source node. Initially, the arborescence $T$ contains only the source node. At each iterative step, the arborescence $T$ is grown by one path from $T$ with least total power that can reach a destination not yet in $T$. This path can be found by collapsing the entire arborescence $T$ into one artificial node and then applying the single-source shortest-path algorithm. This procedure is repeated until all required nodes are included in $T$. This heuristic is referred to as Shortest Path First (SPF). It can be regarded as an adaptation of MST for Min-Power Asymmetric Broadcast Routing. Indeed, when the communication session is a broadcast, it acts the same way as MST. From Theorem 5, we have the following performance of SPF.

Corollary 6: The approximation ratio of SPF is between 6 and $2 c$, which is at most 24 .

In the next, we present an adaptation of BIP for Min-Power Asymmetric Broadcast Routing to a heuristic, which we refer to as Minimum Incremental Path First (MIPF), for Min-Power Asymmetric Multicast Routing. MIPF is implemented as follows. Throughout the execution we maintain an arborescence $T$ rooted at the source node. Initially, the arborescence $T$ contains only the source node. At each iterative step, we first find a path from $T$ with the least incremental power that can reach a destination not yet in $T$, where the incremental power of a path is defined as its total power minus the transmission power in $T$ of its first node. To find this path, we contract $T$ into an artificial node $t$ and construct a weighted complete graph over $t$ and the rest nodes not in $T$ as follows. The weight of the edge between any pair of nodes $u_{1}, u_{2} \notin T$ is still $\left\|u_{1} u_{2}\right\|^{\kappa}$. The weight of an edge between a node $u \notin T$ and the node $t$ is given by

$$
\max \left\{0, \min _{v \in T}\left(\|u v\|^{\kappa}-p_{T}(v)\right)\right\}
$$

where $p_{T}(v)$ is the transmission power in $T$ of node $v$. We then apply the single-source shortest-path algorithm on this constructed graph to find the shortest paths from $t$ to all destination nodes not in $T$ and pick up the one which has the smallest weight. The desired path is then obtained by replacing $t$ with the appropriate node in $T$. After this path is found, the entire path is attached to $T$. This procedure is repeated until all destinations are included in $T$.

Note that if the given communication session is a broadcast, the algorithm MIPF acts in the same way as BIP. As the approximation ratio of BIP for broadcasting is at least (13/3) [15], so must be the approximation ratio of MIPF for multicasting. In the next, we will derive upper bounds on its approximation ratio.

Theorem 7: The approximation ratio of MIPF is between $(13 / 3)$ and $2 c$, which is at most 24.

Proof: Fix a communication session $(s, D)$ and let opt be the minimum power required by its asymmetric routing. Let $T^{*}$ be a Steiner minimum tree for $\{s\} \cup D$ in $G$, and $T$ be the Steiner tree for $\{s\} \cup D$ in $G$ output by the algorithm $\mathcal{A}$.
Let $H$ be the weighted complete graph over $\{s\} \cup D$ in which the weight of each edge is equal to the weight of the shortest-path in $G$ between the endpoints of this edge. Let $\operatorname{mst}(H)$ denote the minimum spanning tree of $H$. The weight of $\operatorname{mst}(H)$ in $H$ is exactly the weight of the Steiner tree for $\{s\} \cup D$ in $G$ produced by Takahashi-Matsuyama Steiner tree heuristic [14]. Thus, the weight of $\operatorname{mst}(H)$ in $H$ is at most $2 \omega\left(T^{*}\right)$.

Now we build another weighted complete graph $H^{\prime}$ over $\{s\} \cup D$ as follows. Suppose that during the execution of MIPF the nodes in $\{s\} \cup D$ are added in the order $u_{0}, u_{1}, \ldots, u_{m}$ where $u_{0}=s$. Let $T_{i}$ be the arborescence just after node $u_{i}$ is added to $T$. In $H^{\prime}$, the weight of the edge $u_{i} u_{i+1}$ is equal to the incremental power of the path from $T_{i}$ to $u_{i+1}$; and the weight of any other edge is the same as that in $H$. Then $H^{\prime}$ has the following properties.

1) The weight of any minimum spanning tree in $H^{\prime}$ is no more than the weight of $\operatorname{mst}(H)$ in $H$. This is because each edge $u_{i} u_{i+1}$ has the same or less weight in $H^{\prime}$ than in $H$ and every other edge has the same weight in $H^{\prime}$ as in $H$.

2) The path $u_{0} u_{1} \ldots u_{m}$ is a minimum spanning tree of $H^{\prime}$. Indeed, the execution of Prim's algorithm on $H^{\prime}$ will emulate the algorithm MIPF on $G$ in the sense that it will add the nodes in $\{s\} \cup D$ in the same order.

3) The total power required by $T$ is exactly the weight of the path $u_{0} u_{1} \ldots u_{m}$ in $H^{\prime}$. This can be easily proven by induction on $i$ for $1 \leq i \leq m$.

From these three properties, we conclude that the total power required by $T$ is at most the weight of $\operatorname{mst}(H)$ in $H$, which is in turn at most $2 \omega\left(T^{*}\right)$. The theorem then follows from Lemma 4.

\section{B. Symmetric Multicasting}

In this section, we present constant-approximation algorithms for Min-Power Symmetric Multicast Routing. Let $\mathcal{A}$ be any polynomial-time approximation algorithm for Steiner minimum tree in graphs. For any given communication session $(s, D)$, we apply $\mathcal{A}$ on $G$ to obtain a Steiner tree for $\{s\} \cup D$, which is used for the symmetric routing of $(s, D)$. We use $\mathcal{A}_{s}$ to denote this heuristic for Min-Power Symmetric Multicast Routing. Before we analyze the performance of $\mathcal{A}_{s}$, we introduce some notations. Consider a subgraph $H$ of $G$. For each vertex $v$ in $V(H)$, the power of $v$ determined by $H$ is denoted by

$$
p_{H}(v) \equiv \max _{u \mid u v \in E(H)} \omega(u v)
$$

i.e., the weight of the heaviest edge incident to $v$ in $H$. The power of $H$ is denoted by

$$
p(H) \equiv \sum_{v \in V(H)} p_{H}(v) .
$$

Theorem 8: For any $\rho$-approximation algorithm $\mathcal{A}$ for Steiner minimum tree, the approximation ratio of the algorithm $\mathcal{A}_{s}$ is at most $2 \rho$.

Proof: Fix a communication session $(s, D)$. We use OPT to denote the min-power arborescence for $(s, D)$ with total 
power opt, and $T$ to denote the Steiner tree for $\{s\} \cup D$ in $G$ output by the algorithm $\mathcal{A}$. Let $T^{*}$ be a Steiner minimum tree for $\{s\} \cup D$ in $G$.

We first claim that opt $>\omega\left(T^{*}\right)$. To prove this, we orient OPT to an arborescence rooted at $s$. For each node $v$ of OPT other than the root $s$, let $v^{\prime}$ be its parent in the arborescence. Then

$$
p_{\mathrm{OPT}}(v) \geq \omega\left(v v^{\prime}\right)
$$

This implies that

$$
\begin{aligned}
\text { opt } & =p(\mathrm{OPT})=p_{\mathrm{OPT}}(s)+\sum_{v \in V(\mathrm{OPT}) \backslash\{s\}} p_{\mathrm{OPT}}(v) \\
& \geq p_{\mathrm{OPT}}(s)+\omega(\mathrm{OPT})>\omega(\mathrm{OPT}) \geq \omega\left(T^{*}\right)
\end{aligned}
$$

Next, we claim that $p(T) \leq 2 \omega(T)$. Indeed, by definition, we have

$$
\begin{aligned}
p(T) & =\sum_{v \in V(T)} p_{T}(v)=\sum_{v \in V(T)} \max _{u \mid u v \in E(T)} c(u v) \\
& \leq \sum_{v \in V(T)} \sum_{u \mid u v \in E(T)} \omega(u v) \\
& =2 \sum_{e \in E(T)} \omega(e) \\
& =2 \omega(T) .
\end{aligned}
$$

Therefore

$$
p(T) \leq 2 \omega(T) \leq 2 \rho \cdot \omega\left(T^{*}\right)<2 \rho \cdot \text { opt }
$$

The theorem thus follows.

We remark that Theorem 8 holds for arbitrarily edgeweighted graphs and its proof remains valid for arbitrary edge weights as well.

\section{SUMMARY}

In this paper, we first derive $\Omega(n)$-lower bounds on the approximation ratios of the three heuristics P-MST, P-SPT, and P-BIP proposed by Wieselthier et al. [16] for Min-Power Asymmetric Multicast Routing. Specifically, the approximation ratio of P-SPT is at least $((n-1) / 2)$, the approximation ratio of P-MST is at least $n-1$, and the approximation ratio of P-SPT is at least $n-2-o(1)$. We then present constant-approximation algorithms for Min-Power Asymmetric Multicast Routing. We show that any $\rho$-approximation Steiner tree algorithm gives rise to a $c \rho$-approximation heuristic for Min-Power Asymmetric Multicast Routing, where $c$ is a constant between 6 and 12. In particular, the Takahashi-Matsuyama Steiner tree heuristic [14] leads to SPF, which has an approximation ratio of at most $2 c$. We also present another heuristic MIPF for Min-Power Asymmetric Multicast Routing, and show that its approximation ratio is between $(13 / 3)$ and $2 c$. Both SPF and MIPF can be regarded as an adaptation of MST and BIP, respectively, in a different manner than pruning. Finally, we prove that any $\rho$-approximation Steiner tree algorithm also gives rise to a $2 \rho$-approximation algorithm for Min-Power Symmetric Multicast Routing.

We recognize that approximation ratio is only one of several figures of merit that can be used in evaluating heuristics. While it is useful from the theoretical viewpoint, it may be restrictive from the practical viewpoint. It would be interesting to know how well the average performance of all the heuristics proposed in the literature and in this paper is. However, the study of the average performance requires sophisticated random geometric analysis, such as those conducted in [1]-[17]. We leave such study as future works. Another challenge, and a topic of continued research, is the development of efficient distributed implementation of some of the heuristics proposed in the literature and in this paper.

\section{REFERENCES}

[1] D. Aldous and J. M. Steele, "Asymptotics for Euclidean minimal spanning trees on random points," Probabil. Theory Relat. Fields, vol. 92, pp. 247-258, 1992.

[2] D. M. Blough, M. Leoncini, G. Resta, and P. Santi, "On the symmetric range assignment problem in wireless ad hoc networks," in Proc. 2nd IFIP Int. Conf. Theoretical Computer Science, Montreal, QC, Canada, Aug. 2002, pp. 71-82.

[3] G. Calinescu, I. Mandoiu, and A. Zelikovsky, "Symmetric connectivity with minimum power consumption in radio networks," in Proc. 2nd IFIP Int. Conf. Theoretical Computer Science, Montreal, QC, Canada, Aug. 2002, pp. 119-130.

[4] W. T. Chen and N. F. Huang, "The strongly connecting problem on multihop packet radio networks," IEEE Trans. Commun., vol. 37, pp. 293-295, Oct. 1989.

[5] A. Clementi, P. Crescenzi, P. Penna, G. Rossi, and P. Vocca, "On the complexity of computing minimum energy consumption broadcast subgraphs," in Proc. 18th Annu. Symp. Theoretical Aspects of Computer Science, vol. LNCS 2010, 2001, pp. 121-132.

[6] T. J. Cormen, C. E. Leiserson, and R. L. Rivest, Introduction to Algorithms. New York: MIT Press and McGraw-Hill, 1990.

[7] L. M. Feeney and M. Nilson, "Investigating the energy consumption of a wireless network interface in an ad hoc networking environment," in Proc. 20th IEEE INFOCOM, 2001, pp. 1548-1557.

[8] F. K. Hwang, D. S. Richards, and P. Winter, The Steiner Tree Problem. Amsterdam, The Netherlands: North-Holland, 1992.

[9] A. O. Ivanov and A. A. Tuzhilin, Minimal Networks: The Steiner Problem and Its Generalizations. Cleveland, OH: CRC Press, 1994.

[10] T. S. Rappaport, Wireless Communications: Principles and Practices. Englewood Cliffs, NJ: Prentice-Hall, 1996.

[11] G. Robins and A. Zelikovsky, "Improved Steiner tree approximation in graphs," in Proc. ACM/SIAM Symp. Discrete Algorithms (SODA'2000), pp. 770-779.

[12] S. Singh, C. S. Raghavendra, and J. Stepanek, "Power-aware broadcasting in mobile ad hoc networks," in Proc. IEEE PIMRC'99, Osaka, Japan, Sept. 1999, pp. 22-31.

[13] J. M. Steele, "Growth rates of Euclidean minimal spanning trees with power weighted edges," Ann. Probabil., vol. 16, pp. 1767-1787, 1988.

[14] H. Takahashi and A. Matsuyama, "An approximate solution for the Steiner problem in graphs," Mathematica Japonica, vol. 24, pp. 573-577, 1980.

[15] P.-J. Wan, G. Călinescu, X.-Y. Li, and O. Frieder, "Minimum-energy broadcast in static ad hoc wireless networks," ACM Wireless Networks, vol. 8, no. 6, pp. 607-617, Nov. 2002.

[16] J. E. Wieselthier, G. D. Nguyen, and A. Ephremides, "On the construction of energy-efficient broadcast and multicast trees in wireless networks," in Proc. 19th IEEE INFOCOM, 2000, pp. 585-594.

[17] J. E. Yukich, "Asymptotics for weighted minimal spanning trees on random points," Stoch. Process. Appl., vol. 85, pp. 123-128, 2000. 


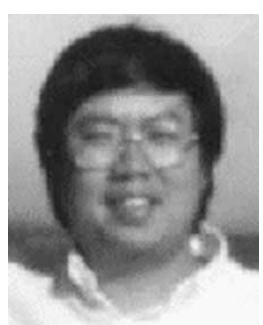

Peng-Jun Wan received the B.S. degree from Qsinghua University, China, the M.S. degree from The Chinese Academy of Science, Beijing, and the Ph.D. degree from the University of Minnesota, Minneapolis.

$\mathrm{He}$ is currently an Associate Professor in computer science at the Illinois Institute of Technology, Chicago. His research interests include wireless networks and optical networks.

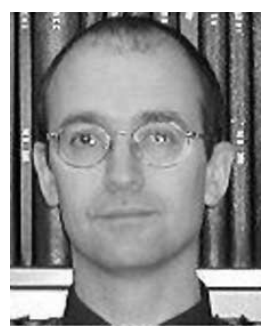

Gruia Călinescu received the Diploma from the University of Bucharest, Hungary, and the Ph.D. degree from the Georgia Insitute of Technology, Atlanta.

He is currently an Assistant Professor of computer science at the Illinois Institute of Technology, Chicago. His research interests are in the area of algorithms.

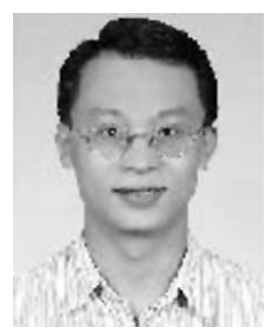

Chih-Wei Yi received the B.S. and M.S. degrees from National Taiwan University. He is currently working toward the Ph.D. degree at the Illinois Institute of Technology, Chicago. His dissertation research focuses on wireless ad hoc networks. 